\title{
Comunidades planctónicas y bacterianas asociadas al cultivo de bocachico Prochilodus magdalenae con tecnología biofloc
}

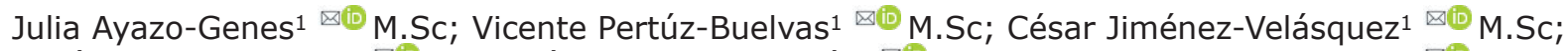

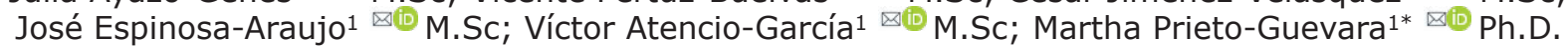

${ }^{1}$ Universidad de Córdoba, Facultad de Medicina Veterinaria y Zootecnia, Departamento de Ciencias Acuícolas, Instituto de Investigación Piscícola-CINPIC, Montería, Colombia.

*Correspondencia: mprieto@correo.unicordoba.edu.co

Recibido: Julio 2018; Aceptado: Noviembre 2018; Publicado: Mayo 2019.

\section{RESUMEN}

Objetivo. Describir las comunidades planctónicas y bacterianas asociadas al cultivo de bocachico Prochilodus magdalenae con tecnología biofloc (BFT). Materiales y métodos. En nueve tanques rectangulares de concreto con volumen útil de $6.0 \mathrm{~m}^{3}$, se sembraron alevinos de bocachico con peso promedio de $1.6 \pm 0.2 \mathrm{~g}$, a tres densidades 5 (T1), 10 (T2) y 20 (T3) peces $/ \mathrm{m}^{3}$ con BFT, durante 120 días de cultivo. La identificación y cuantificación de los microorganismos se realizó cada ocho días, en una muestra de $250 \mathrm{ml}$ de agua por tanque, mediante análisis de alícuotas en cámaras Sedgwick-Rafter y/o Neubauer bajo microscopio a 10x y 40x. Los días 15, 45 y 90 del cultivo se caracterizaron las comunidades bacterianas tomando una muestra de $2 \mathrm{~g}$ de floc en $90 \mathrm{ml}$ de solución salina estéril y sometidas a pruebas microbiológicas convencionales. Resultados. Se identificaron cinco grupos planctónicos (microalgas, rotíferos, cladóceros, copépodos y protistas con predominancia de ciliados) con mayor cantidad de rotíferos y protistas en los cultivos con menor densidad ( $\mathrm{T} 1$ y $\mathrm{T} 2$ ); y la mayor afluencia de microorganismos osciló entre $174.9 \pm 21.4 \mathrm{ind} / \mathrm{ml}$ (T1) y $125.6 \pm 16.1 \mathrm{ind} / \mathrm{ml}$ (T2). En el grupo de bacterias fue posible identificar 10 cepas: Escherichia coli, Enterobacter sp., Klebsiella sp., Salmonella sp. (Enterobacteriaceae) Bacillus subtilis, Bacillus sp, Lactobacillus sp, Pseduodomonas sp (Vibrionaceae), Micrococcus sp, Staphylococcus sp (Cocos gram+). Conclusiones. La composición del plancton fue similar en todos los tratamientos, con rotífero y protistas como los más abundantes; la mayor proporción de bacterias fueron Enterobacterias y Heterotróficas.

Palabras clave: Bacterias, ciliados, rotíferos, zooplancton (Fuente: UNESCO, CAB).

\section{ABSTRACT}

Objective. To describe the planktonic communities and bacteria associated with the fish culture of Bocachico Prochilodus magdalenae with biofloc technology (BFT). Materials and methods. bocachico fingerlings, with an average weight of $1.6 \pm 0.2 \mathrm{~g}$, were stocked to three densities 5 (T1), 10 (T2) and 20 (T3) fish/ $\mathrm{m}^{3}$ with BFT, in nine rectangular concrete tanks with $6.0 \mathrm{~m}^{3}$ of volume, during 120 days of culture. The identification and quantification of the microorganisms was performed every 8 days in a sample of $250 \mathrm{ml}$ of water per tank, by analyzing aliquots on a Sedgwick-Rafter and/or Neubauer chambers under a microscope at 10x and 40x magnification. The days 15, 45 and 90 of fish culture, bacterial communities were characterized taking a sample of $2 \mathrm{~g}$ of floc in $90 \mathrm{ml}$ of sterile saline solution and subjected to conventional microbiological tests. Results. Planktonic five groups (microalgae, rotifers, cladocerans, copepods and protists with ciliates predominant) with more rotifers and protists in the fish cultures with lower density (T1 and T2) were identified; and the highest amount of microorganisms osciled between $174.9 \pm 21.4 \mathrm{ind} / \mathrm{ml}$ (T1) and $125.6 \pm 16.1$ ind/ml (T2). In the bacteria group was possible to identify 10 bacterial strains: Escherichia coli, Enterobacter sp., Klebsiella sp., Salmonella sp. (Enterobacteriaceae) Bacillus subtilis, Bacillus sp, Lactobacillus $s p$, Pseduodomonas sp (Vibrionaceae), Micrococcus sp, Staphylococcus $s p$ (Cocus gram+). Conclusion. The composition of plankton was similar in all treatments, with rotifers and protists as the most abundant; bacteria showed a higher proportion between Enterobacteria and Heterotrophic.

Keywords: Bacteria, ciliates, rotifers, zooplankton (Source: UNESCO, CAB).

Como citar (Vancouver)

Ayazo-Genes ], Pertúz-Buelvas V, Jiménez-Velásquez C, Espinosa-Araujo J, Atencio-García V, Prieto-Guevara M. Comunidades planctónicas y bacterianas asociadas al cultivo de bocachico Prochilodus magdalenae con tecnología biofloc. Rev MVZ Cordoba. 2019; 24(2):7209-7217. DOI: https://doi.org/10.21897/rmvz.1648

(CEI (los) autor (es), Revista MVZ Córdoba 2019. Este artículo se distribuye bajo los términos de la licencia internacional Creative Commons Attribution 4.0 (https://creativecommons.org/licenses/by-sa/4.0/), que permite el uso sin restricciones, la distribución y la reproducción en cualquier medio, siempre que se otorgue el crédito apropiado al autor o autores originales y la fuente. 


\section{INTRODUCCIÓN}

La tecnología de cultivo biofloc (biofloc technology o BFT), es un concepto basado en la manipulación de la comunidad microbiana bajo condiciones controladas en el sistema de cultivo, facilitando la producción de los animales acuáticos de manera sostenible y biosegura (1). Esta tecnología descansa en las relaciones de óxidoreducción del ciclo del nitrógeno y la adición de sustratos ricos en carbono (melaza, harina de yuca, glicerol, entre otros) para el secuestro y reciclaje del nitrógeno en forma de proteína microbiana (2).

Estas condiciones favorecen el desarrollo de una comunidad microbiana benéfica, asentada en pequeños flóculos de materia orgánica continuamente resuspendidos en la columna de agua por la acción de la aireación permanente que reciclan el nitrógeno del amonio producido por los peces en la síntesis de proteína unicelular; la cual puede ser ingerida y asimilada por los peces, a través de actividades de pastoreo sobre las comunidades de microorganismos planctónicos asociados a los flóculos, posibilitando la utilización de dietas bajas en proteínas en comparación con sistemas convencionales de cultivo $(3,4,5)$.

La transformación de la materia orgánica particulada y los organismos en la cadena trófica microbiana, se han propuesto como posibles fuentes de alimentos en los cultivos con BFT (5), a partir de la compleja interacción en toda la columna de agua, entre la materia orgánica, el sustrato físico y una amplia gama de microorganismos como microalgas, bacterias libres y adheridas, agregados de la materia orgánica y herbívoros tales como rotíferos, ciliados, protozoos flagelados y copépodos (6).

Como resultado de esta interacción, se establece el reciclado continuo de compuestos nitrogenados del sistema, además de la disponibilidad de una rica fuente natural de proteína y lípido a disposición in situ las 24 horas del día, como fuente de alimento para las especies cultivadas $(5,7,8)$. La asimilación de esta fuente de alimentación, dependerá en gran medida del hábito alimenticio de la especie, condiciones del sistema y de las poblaciones de microorganismos que predominen. En el caso de especies omnívoras como tilapia $(5,9)$ y camarón existe una amplia aplicabilidad de esta tecnología para su cultivo $(8,10,11)$; pero no para especies detritívoras como bocachico Prochilodus magdalenae.

Dada la importancia de la productividad natural en el reciclaje de nutrientes y mantenimiento de la calidad del agua, es necesario conocer la composición microbiológica adherida al floc para ofrecer un manejo adecuado, maximizar sus efectos benéficos como la remoción de compuestos nitrogenados y la alimentación de los animales en cultivo (12). Sin embargo; es poca la información disponible acerca de la caracterización de los microorganismos presentes en sistemas biofloc, para entender el papel desempeñado por la biomasa microbiana, además de las referencias de la aplicabilidad de esta tecnología en especies nativas de importancia comercial.

Por lo anterior, y en vista de la necesidad de implementar sistemas intensivos de producciones piscícolas ambientalmente sostenibles y económicamente rentables, el presente trabajo caracteriza las comunidades planctónicas y bacterianas presentes en el cultivo intensivo de bocachico en fase de preengorde a tres densidades de siembra con tecnología biofloc.

\section{METERIALES Y MÉTODOS}

Sitio de estudio. El estudio se realizó en el Instituto de Investigación Piscícola de la Universidad de CórdobaCINPIC (Montería, Córdoba, Colombia). En nueve tanques rectangulares de concreto de $6.0 \mathrm{~m}^{3}$ de volumen útil, se sembraron 630 alevinos de bocachico con $1.6 \pm 0.5 \mathrm{~g}$ de peso promedio a tres densidades de siembra 5 (T1), 10 (T2), 20 (T3) peces $/ \mathrm{m}^{3}$, para caracterizar las comunidades planctónicas y microbiota asociada al cultivo de bocachico, con tecnología biofloc, en la fase de preengorde, durante 120 días de cultivo. El cultivo fue mantenido con aireación permanente suministrada con un blower de $1.5 \mathrm{HP}$ y manguera polidifusora, cubiertos con malla polisombra para reducir la entrada de la luz y protección contra predadores.

Inóculo inicial de floc. El inóculo inicial del sistema biofloc fue establecido con agua de un estanque de uso piscícola excavado en tierra con presencia de bacterias autótrofas y heterótrofas; y para el fomento y desarrollo de éstas últimas se adicionó melaza, como fuente de carbono, con una relación C: N cercana a 20:1. Para la aplicación de la melaza se utilizó la fórmula: Melaza $=N^{*}(20-12)$, donde, 20 es la relación teórica de C:N (20:1) en el manejo de cultivo BFT y 12 es la relación C:N (12:1) de las raciones alimenticias de 24\% proteína bruta (2) y NT corresponde a la cantidad total de nitrógeno en el cultivo, estimado con la ecuación propuesta por Kubitza (13) NT= [(cantidad de $\mathrm{N}-\mathrm{NH}_{3}+$ $\mathrm{N}-\mathrm{NH}_{4}{ }^{+}+$cantidad de $\left.\mathrm{N}-\mathrm{NO}_{2}{ }^{-}\right)^{*}$ volumen de agua]/1000.

Posterior a la estabilización del inóculo inicial (14 días), basados en el manejo de los compuestos nitrogenados y la caracterización cualitativa y cuantitativa de los microorganismos presentes en el inóculo, se procedió a transferir $600 \mathrm{~L}$ de inóculo en cada unidad experimental, seguidamente se completaron hasta $6.0 \mathrm{~m}^{3}$ utilizando agua del sistema de distribución del CINPIC.

La calidad del agua se evaluó durante los periodos de estabilización y cultivo. Dos veces al día se tomaron registros del oxígeno disuelto (OD), $\mathrm{pH}$ y temperatura, con ayuda de un oxímetro (YSI, 550A, Usa) y un pHmetro (YSI, pH100, Usa). El amonio total, nitritos y nitratos, se midieron cada dos días; la dureza total y alcalinidad total, cada ocho días con ayuda de un fotómetro (YSI, 9500, Usa).

Caracterización de las comunidades planctónicas. Semanalmente se tomaron cinco muestras de floc con un beaker de $50 \mathrm{ml}$ en cinco puntos distintos de cada unidad de cultivo, homogenizadas en un Erlenmeyer de $250 \mathrm{ml}$ y fijadas en formol buferado $10 \%$; luego, submuestras de $1 \mathrm{ml}$ por triplicado fueron tomadas para su identificación y cuantificación de los microorganismos asociados a los flóculos. Las muestras fueron analizadas en cámaras Segwick-Rafter y/o Neubauer con ayuda de un microscopio óptico (Carl Zeiss, Axiostar, Alemania) y un microscopio invertido de contraste de fase positiva (Carl Zeiss, Primo Vert, Alemania), con objetivos entre 10x y 40x y un analizador de imágenes (Carl Zeiss, Axiovision 4, Alemania). La identificación de especies de los diferentes grupos del plancton se realizó con ayuda de claves taxonómicas entre ellas las de Aladro-Lubel et al (14). 
La abundancia de microorganismos (A) por ítem identificado, se estimó con la fórmula: $A=((\mathrm{Vcf})(\mathrm{Ni})) /$ (Vti) Vc; dónde, Vcf corresponde al volumen de la concentración; $\mathrm{Ni}$ al número de individuos contados; $\mathrm{Vti}$ al volumen total inicial; $\mathrm{Vc}$ al volumen de la muestra contada. La abundancia fue expresada en número de individuos $/ \mathrm{mL}$. La relación entre el número de especies y su abundancia relativa en tiempo y espacio, se interpretó a través de los conteos semanales realizados por tratamiento. Además, se estimaron tres índices ecológicos para expresar la diversidad de especies (índice de Shannon-Wiener, $\mathrm{H}^{\prime}$ ), la uniformidad (índice Pielou, J') y la dominancia de los organismos (índice de Simpson, $\lambda$ ).

Aislamiento y caracterización bacteriana. La caracterización de las comunidades bacterianas se realizó según el método descrito por Monroy-Dosta et al (15). A partir de la formación de los pequeños flóculos en el inóculo inicial y a los 15,45 y 90 días de cultivo, se tomaron muestras de $2 \mathrm{~g}$ que se inocularon en 90 $\mathrm{ml}$ de solución salina estéril, se realizaron sucesivas diluciones $(1: 10)$ y se sembró $0,1 \mathrm{ml}$ de las mismas por triplicado en cajas de agar MSR (Man-Rogosa Sharpe), BHI (Infusión Cerebro-Corazón), TCBS (TiosulfatoCitrato-Sales Biliares) y TSA (Tripticasa-soja). Las placas se incubaron a $27^{\circ} \mathrm{C}$ durante $24 \mathrm{~h}$ y luego se efectuó el conteo de las unidades formadoras de colonias (UFC/ml); posteriormente, a través de resiembras sucesivas, las cepas fueron purificadas. La tinción de Gram se utilizó para observar la morfología celular con ayuda de un microscopio óptico de luz (Carl Zeiss, Axiostar, Alemania). Pruebas microbiológicas convencionales fueron realizadas a las cepas aisladas para su identificación como catalasa, oxidasa, óxido fermentación, movilidad e indol. Finalmente, se confirmó la identificación de las cepas con el sistema API20E, API20NE, APICHL, APICHL50. Además de las muestras de bacterias tomadas en la columna de agua; al final del cultivo, de cada unidad experimental con una malla de $100 \mu \mathrm{m}$, fueron tomadas para su caracterización una muestra de lodo del fondo generado por la dinámica de cultivo.

Análisis estadístico. El estudio se realizó bajo un diseño completamente al azar (DCA); las variables fueron sometidas a pruebas de normalidad (test de Shapiro-Wilk) y homocedasticidad (test de Bartlett); posteriormente se aplicó análisis de varianza (ANOVA) seguido de una prueba de rango múltiple de Duncan. En todos los casos, se utilizó un nivel de confianza del $95 \%$. El análisis estadístico se realizó con el software SAS versión para Windows 9.2 (SAS Institute Inc, Cary, NC, USA).

\section{RESULTADOS}

Los parámetros de calidad de agua durante el cultivo de bocachico en sistema biofloc se presenta en la tabla 1. El oxígeno disuelto presentó promedios superiores a $6.0 \mathrm{mg} / \mathrm{l}$; la temperatura osciló entre $28.6 \pm 0.3^{\circ} \mathrm{C}$ (T1) y $28.4 \pm 0.3^{\circ} \mathrm{C}(\mathrm{T} 3)$; el $\mathrm{pH}$ se mantuvo en promedio en 8.0 en los tres tratamientos; la dureza osciló entre $101.9 \pm 67.8 \mathrm{mg} / \mathrm{l} \mathrm{CaCO}_{3}$ (T2) y $90.2 \pm 52.4 \mathrm{mg} / \mathrm{l} \mathrm{CaCO}_{3}$ (T1), sin presentar diferencia significativa entre los tratamientos $(p>0.05)$. La alcalinidad fue mayor significativamente $(p<0.05)$ en los tratamientos con las más altas densidades $\left(\mathrm{T} 2=114.5 \pm 57.9 \mathrm{mg} / \mathrm{CaCO}_{3}\right.$,
$\left.\mathrm{T} 3=101.9 \pm 67.8 \mathrm{mg} / \mathrm{l} \mathrm{CaCO}_{3}\right)$. Los mayores promedios de TAN $(5.1 \pm 5.3 \mathrm{mg} / \mathrm{l})$ y $\mathrm{NH}_{3}(2.3 \pm 2.4 \mathrm{mg} / \mathrm{l})$ se registraron en $\mathrm{T} 3$, observándose diferencia estadística con los otros tratamientos $(p<0.05)$. Los valores de $\mathrm{NO}_{2}^{-}$, se mantuvieron en promedio en $0.7 \mathrm{mg} / \mathrm{L}$ en todos los tratamientos; los valores promedios de $\mathrm{NO}_{3}$ fueron significativamente mayores $(p<0.05)$ en los tratamientos con más altas densidades $(T 2=19.2 \pm 12.3$ $\mathrm{mg} / \mathrm{l}, \mathrm{T} 3=19.2 \pm 2.2 \mathrm{mg} / \mathrm{l})(\mathrm{p}>0.05)$.

Tabla 1. Parámetros de calidad de agua durante el cultivo de $P$. magdalenae en fase de preengorde con tecnología biofloc. Letras diferentes en la misma fila, indica diferencia significativa $(p<0.05)$.

\begin{tabular}{|c|c|c|c|}
\hline & T1 & T2 & T3 \\
\hline Temperatura $\left({ }^{\circ} \mathrm{C}\right)$ & $28.6 \pm 0.3^{a}$ & $28.5 \pm 0.3^{a}$ & $28.4 \pm 0.3^{a}$ \\
\hline $\mathrm{OD}(\mathrm{mg} / \mathrm{l})$ & $6.9 \pm 0.3^{a}$ & $6.9 \pm 0.2^{a}$ & $6.8 \pm 0.3^{a}$ \\
\hline $\mathrm{pH}$ & $8.0 \pm 0.3^{a}$ & $8.0 \pm 0.1^{a}$ & $8.0 \pm 0.2^{a}$ \\
\hline Alcalinidad $\left(\mathrm{mg} / \mathrm{l} \mathrm{CaCO}{ }_{3}\right)$ & $95.3 \pm 44.5^{b}$ & $114.5 \pm 57.9^{a}$ & $108.6 \pm 63.2^{a}$ \\
\hline Dureza $\left.(\mathrm{mg} / \mathrm{I} \mathrm{CaCO})_{3}\right)$ & $90.2 \pm 52.4^{a}$ & $101.9 \pm 67.8^{a}$ & $101.5 \pm 59.0^{a}$ \\
\hline TAN (mg/l) & $2.6 \pm 1.1^{c}$ & $3.3 \pm 2.1^{b}$ & $5.1 \pm 5.3^{a}$ \\
\hline $\mathrm{NH}_{3}(\mathrm{mg} / \mathrm{l})$ & $1.2 \pm 0.5^{b}$ & $1.5 \pm 1.0^{b}$ & $2.3 \pm 2.4^{a}$ \\
\hline $\mathrm{NO}_{2}^{-}(\mathrm{mg} / \mathrm{l})$ & $0.7 \pm 0.5^{a}$ & $0.7 \pm 0.6^{a}$ & $0.7 \pm 0.5^{a}$ \\
\hline $\mathrm{NO}_{3}^{-}(\mathrm{mg} / \mathrm{l})$ & $16.4 \pm 11.2^{b}$ & $19.2 \pm 12.3^{a}$ & $19.2 \pm 2.2^{a}$ \\
\hline
\end{tabular}

Microorganismos planctónicos durante la estabilización y maduración del inóculo de floc. Los grupos de microorganismos identificados durante el periodo de estabilización y maduración del inóculo de floc se relacionan en la tabla 2 . Los copépodos fueron los más abundantes $(13.0 \pm 0.0$ ind $/ \mathrm{ml})$ mostrando diferencia estadística con los otros grupos identificados $(p<0.05)$. Los rotíferos y las microalgas fueron los grupos con mayor diversidad de especies. En los rotíferos las especies Lecane luna (21.0 $\pm 4.4 \mathrm{ind} / \mathrm{ml}$ ) y Brachionus havanaensis $(17.0 \pm 2.6 \mathrm{ind} / \mathrm{ml})$ fueron las más abundantes, mientras que para las microalgas predominaron Scenedesmus securiformis $(5.7 \pm 2.1)$ y Pinnularia sp $(4.0 \pm 1.0)$.

Caracterización de los microorganismos planctónicos a diferentes densidades de cultivo del bocachico. Los grupos del zooplancton identificados durante el periodo de cultivo en la fase de preengorde con tecnología biofloc se muestran en la tabla 3. La mayor abundancia de zooplancton se presentó en T1 $(174.9 \pm 21.4$ ind $/ \mathrm{ml})$ y la menor en T3 (121.3 \pm 15.9 ind $/ \mathrm{ml}$ ) observándose diferencia estadística entre estos valores $(p<0.05)$. A la menor densidad de cultivo (T1) se registró mayor abundancia de rotíferos $(35.6 \pm 18.6 \mathrm{ind} / \mathrm{ml})$, protistas $(69.6 \pm 28.6 \mathrm{ind} /$

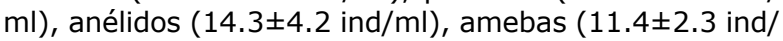
$\mathrm{ml}$ ) y cladóceros $(6.6 \pm 2.2 \mathrm{ind} / \mathrm{ml})$ observándose diferencia estadística entre densidades de cultivo $(p<0.05)$. En T1 $(18.8 \pm 10.2 \mathrm{ind} / \mathrm{ml})$ y T3 $(13.9 \pm 12.1 \mathrm{ind} / \mathrm{ml})$ se registró la mayor abundancia de ciliados; sin diferencia estadísticas entre estos valores $(p>0.05)$. El grupo de los copépodos no mostró diferencia significativa entre los tratamientos ( $p>0.05)$ oscilando entre $5.2 \pm 1.5 \mathrm{ind} / \mathrm{ml}$ (T3) y $(6.7 \pm 1.5$ ind $/ \mathrm{ml})$.

Entre los rotíferos se destacó la abundancia de Euchlanis sp., Habrotrocha lata, Habrotrocha sp., Lecane luna, Lecane sp., Mytilina sp., y Mytilina videns; entre ciliados las morfoespecies más abundantes fueron Vorticellas sp. y Paramecio sp. Los protistas abundantes fueron Arcella vulgaris, Euglypha acanthophora, Euglypha alveolata, Euglypha sp., y Nebela sp. 
Tabla 2. Microorganismos identificados durante la estabilización y maduración del inóculo de floc, previo al inicio de cultivo de $P$. magdalenae en fase de preengorde con tecnología biofloc.

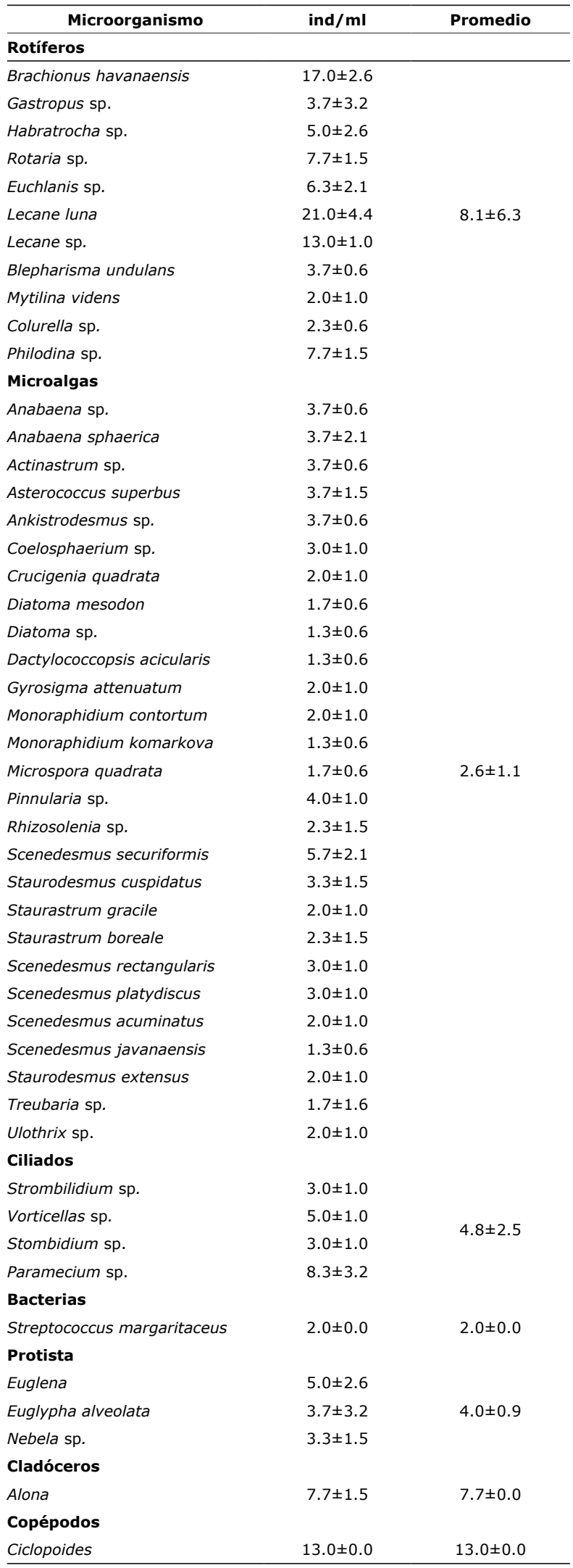

Tabla 3. Abundancia de zooplancton en el cultivo de $P$. magdalenae con tecnología biofloc.

\begin{tabular}{|c|c|c|c|}
\hline \multirow{2}{*}{ Microorganismo } & \multicolumn{3}{|c|}{ Ind $/ \mathrm{ml}$} \\
\hline & T1 & T2 & T3 \\
\hline \multicolumn{4}{|l|}{ Rotíferos } \\
\hline Blepharisma undulans & $20.0 \pm 5.2^{a}$ & & $14.0 \pm 3.2^{a}$ \\
\hline Brachionus havanaensis & $14.0 \pm 0.5^{a}$ & $5.5 \pm 0.3^{b}$ & $7.0 \pm 0.4^{b}$ \\
\hline Colurella sp. & $24.2 \pm 1.5^{a}$ & $7.0 \pm 2.0^{c}$ & $13.7 \pm 8.0^{b}$ \\
\hline Colurella uncinata & $14.0 \pm 3.6^{b}$ & $19.0 \pm 8.2^{a}$ & $27.0 \pm 5.2^{a}$ \\
\hline Euchlanis sp. & $42.5 \pm 18.5^{a}$ & $32.7 \pm 20.1^{b}$ & $30.1 \pm 20.3^{b}$ \\
\hline Gastropus sp. & $3.7 \pm 0.5^{a}$ & $5.5 \pm 0.2^{a}$ & $4.3 \pm 1.0^{a}$ \\
\hline Habrotrocha lata & $52.5 \pm 25.3^{a}$ & $47.1 \pm 28.2^{a}$ & $42.8 \pm 22.0^{a}$ \\
\hline Habrotrocha sp. & $33.5 \pm 23.5^{b}$ & $21.4 \pm 16.3^{b}$ & $53.0 \pm 32.1^{a}$ \\
\hline Keratella sp. & $2.0 \pm 0.2^{b}$ & & $9.5 \pm 1.0^{a}$ \\
\hline Lecane luna & $72.2 \pm 25.6^{a}$ & $57.8 \pm 32.4^{b}$ & $45.2 \pm 25.3^{b}$ \\
\hline Lecane sp. & $53.2 \pm 16.5^{a}$ & $29.3 \pm 12.6^{b}$ & $33.7 \pm 19.5^{b}$ \\
\hline Mytilina sp. & $150.0 \pm 39.5^{a}$ & & $10.0 \pm 2.0^{b}$ \\
\hline Mytilina videns & $41.6 \pm 21.0^{b}$ & $48.7 \pm 18.3^{a}$ & $37.7 \pm 14.3^{b}$ \\
\hline Philodina sp. & $9.1 \pm 0.5^{b}$ & $4.2 \pm 0.3^{c}$ & $14.6 \pm 0.4^{a}$ \\
\hline Polyarthra sp. & & & $2.0 \pm 0.0^{a}$ \\
\hline \multirow[t]{2}{*}{ Rotaria sp. } & $1.0 \pm 0.0^{b}$ & $2.5 \pm 0.0^{a}$ & $2.3 \pm 0.5^{a}$ \\
\hline & $35.6 \pm 18.6^{a}$ & $23.4 \pm 19.6^{b}$ & $21.7 \pm 16.8^{b}$ \\
\hline \multicolumn{4}{|l|}{ Ciliados } \\
\hline Strombilidium sp. & $24.0 \pm 12.6^{a}$ & $8.0 \pm 1.0^{b}$ & $5.0 \pm 2.0^{b}$ \\
\hline Vorticellas sp. & $37.0 \pm 29.4^{a}$ & $17.8 \pm 15.3^{b}$ & $21.3 \pm 15.4^{b}$ \\
\hline Stombidium sp. & $11.0 \pm 0.5^{a}$ & $4.0 \pm 0.5^{b}$ & $13.0 \pm 2.5^{a}$ \\
\hline Euplotes charon & $2.0 \pm 1.0^{b}$ & $12.0 \pm 2.8^{a}$ & \\
\hline Euplotes patella & $1.0 \pm 1.0^{b}$ & $5.1 \pm 2.2^{a}$ & $2.3 \pm 1.2^{b}$ \\
\hline \multirow[t]{2}{*}{ Paramecio sp. } & $37.7 \pm 22.5^{a}$ & $20.0 \pm 16.2^{b}$ & $28.1 \pm 14.6^{a}$ \\
\hline & $18.8 \pm 10.2^{a}$ & $11.1 \pm 8.0^{b}$ & $13.9 \pm 12.1 \mathrm{a}$ \\
\hline \multicolumn{4}{|l|}{ Protista } \\
\hline Arcella magastoma & $40.0 \pm 2.0^{a}$ & & \\
\hline Arcella sp. & $25.5 \pm 2.5^{b}$ & $34.0 \pm 2.5^{a}$ & $29.3 \pm 2.5^{b}$ \\
\hline Arcella vulgaris & $60.0 \pm 22.4^{b}$ & $79.5 \pm 31.8^{a}$ & $71.0 \pm 28.6^{a}$ \\
\hline Centropyxis aculeata & $38.0 \pm 12.8^{a}$ & $21.7 \pm 14.6^{b}$ & $27.0 \pm 12.2^{a}$ \\
\hline Euglena sp. & $57.2 \pm 18.4^{a}$ & $56.9 \pm 16.4^{a}$ & $40.0 \pm 22.6^{b}$ \\
\hline Euglypha acanthophora & $72.3 \pm 32.1^{a}$ & $59.5 \pm 28.6^{b}$ & $77.5 \pm 36.4^{a}$ \\
\hline Euglypha alveolata & $106.0 \pm 36.2^{a}$ & $81.3 \pm 24.8^{b}$ & $84.0 \pm 41.2^{b}$ \\
\hline Euglypha sp. & $130.0 \pm 45.2^{a}$ & $4.0 \pm 1.0^{c}$ & $16.0 \pm 4.3^{b}$ \\
\hline \multirow[t]{2}{*}{ Nebela sp. } & $97.2 \pm 36.2^{a}$ & $77.5 \pm 28.6^{b}$ & $77.6 \pm 30.2^{b}$ \\
\hline & $69.6 \pm 28.6^{a}$ & $51.8 \pm 31.4^{b}$ & $52.8 \pm 24.1^{b}$ \\
\hline \multicolumn{4}{|l|}{ Anélidos } \\
\hline Monhytera similis & $18.7 \pm 5.6^{a}$ & $7.2 \pm 2.6^{b}$ & $7.0 \pm 1.8^{b}$ \\
\hline \multirow[t]{2}{*}{ Aeleosoma sp. } & $9.9 \pm 2.1^{a}$ & $9.5 \pm 3.2^{a}$ & $11.5 \pm 1.5^{a}$ \\
\hline & $14.3 \pm 4.2^{a}$ & $8.4 \pm 3.4^{b}$ & $9.3 \pm 2.1^{b}$ \\
\hline \multicolumn{4}{|l|}{ Amebas } \\
\hline Amebas sp. & $14.0 \pm 2.8^{a}$ & & \\
\hline \multirow[t]{2}{*}{ Astramoeba radiosa } & $8.8 \pm 2.4^{a}$ & $6.3 \pm 2.1^{a}$ & $5.5 \pm 2.8^{a}$ \\
\hline & $11.4 \pm 2.3^{a}$ & $6.3 \pm 1.6^{b}$ & $5.5 \pm 1.5^{b}$ \\
\hline \multicolumn{4}{|l|}{ Cladóceros } \\
\hline Moina sp. & $6.8 \pm 1.8^{a}$ & $5.6 \pm 2.1^{a}$ & $5.2 \pm 1.8^{a}$ \\
\hline \multirow[t]{2}{*}{ Alona sp. } & $6.4 \pm 2.2^{a}$ & $3.9 \pm 2.5^{b}$ & $3.0 \pm 1.8^{b}$ \\
\hline & $6.6 \pm 2.2^{a}$ & $4.8 \pm 2.2^{b}$ & $4.1 \pm 2.8^{b}$ \\
\hline \multicolumn{4}{|l|}{ Copépodos } \\
\hline Ciclopoides & $6.7 \pm 1.5^{a}$ & $5.7 \pm 2.2^{a}$ & $5.2 \pm 1.5^{a}$ \\
\hline Total & \multicolumn{3}{|c|}{$174.9 \pm 21.4^{a} \quad 125.6 \pm 16.1^{b} \quad 121.3 \pm 15.9^{b}$} \\
\hline
\end{tabular}

Letras diferentes en la misma fila, indica diferencia significativa $(p<0.05)$. 
Ayazo-Genes et al - Comunidades y bacterias asociadas al cultivo de peces Prochilodus magdalenae.

En la tabla 4 se muestra la abundancia de las especies y morfoespecies del fitoplancton identificadas. Se destaca la abundancia de Ankistrodesmus acicularis (31.5 \pm 8.0 $\mathrm{cel} / \mathrm{ml} \mathrm{T1})$, Ankistrodesmus sp. (25.5 $\pm 6.4 \mathrm{cel} / \mathrm{ml} \mathrm{T3})$, Crucigenia quadrata $(50.5 \pm 2.0 \mathrm{cel} / \mathrm{ml})$, Monoraphidium komarkova $(50.9 \pm 22.3 \mathrm{cel} / \mathrm{ml} \mathrm{T3})$, Scenedesmus securiformis $(30.2 \pm 10.2 \mathrm{cel} / \mathrm{ml}$ T1) observándose diferencia estadística entre tratamientos $(p<0.05)$.

Tabla 4. Abundancia de especies de fitoplancton (cel/ml), identificados durante el cultivo de $P$. magdalenae con tecnologia biofloc (Valores promedio \pm SD).

\begin{tabular}{|c|c|c|c|}
\hline Microalgas & T1 & T2 & T3 \\
\hline Actinastrum sp. & $1.5 \pm 0.5^{b}$ & $8.0 \pm 1.0^{a}$ & $1.0 \pm 1.0^{b}$ \\
\hline Anabaena sp. & $2.0 \pm 0.3^{c}$ & $4.0 \pm 0.3^{b}$ & $10.7 \pm 1.2^{a}$ \\
\hline Anabaena sphaerica & $6.0 \pm 0.5^{b}$ & $1.0 \pm 0.0^{c}$ & $13.7 \pm 2.5^{a}$ \\
\hline Ankistrodesmus acicularis & $31.5 \pm 8.0^{a}$ & $11.0 \pm 3.0^{b}$ & \\
\hline Ankistrodesmus sp. & $18.5 \pm 8.0^{a}$ & $20.4 \pm 5.0^{a}$ & $25.5 \pm 6.4^{a}$ \\
\hline Asterococcus superbus & $2.0 \pm 0.0^{a}$ & $1.0 \pm 0.0^{a}$ & $1.0 \pm 0.0^{a}$ \\
\hline Coelosphaerium sp. & $20.9 \pm 0.5^{a}$ & $17.2 \pm 1.0^{a}$ & $18.6 \pm 3.5^{a}$ \\
\hline Crucigenia quadrata & $50.5 \pm 2.0^{a}$ & $50.5 \pm 3.5^{a}$ & $41.6 \pm 12.6^{a}$ \\
\hline Crucigenia tetrapedia & $22.2 \pm 1.0^{b}$ & $10.9 \pm 2.0^{c}$ & $29.5 \pm 3.5^{a}$ \\
\hline Dactylococcopsis acicularis & $5.0 \pm 1.0^{c}$ & $18.8 \pm 2.8^{a}$ & $10.8 \pm 2.5^{b}$ \\
\hline Diatoma mesodon & $3.2 \pm 1.0^{a}$ & $1.7 \pm 1.0^{b}$ & $4.4 \pm 2.0^{a}$ \\
\hline Diatoma sp. & $6.0 \pm 1.0^{a}$ & $2.5 \pm 1.0^{b}$ & $2.0 \pm 0.5^{b}$ \\
\hline Gyrosigma attenuatum & $9.2 \pm 2.0^{a}$ & $2.5 \pm 1.8^{b}$ & $8.0 \pm 3.2^{a}$ \\
\hline Microspora quadrata & $24.5 \pm 12.0^{a}$ & $28.0 \pm 16.5^{a}$ & \\
\hline Monoraphidium contortum & $17.4 \pm 5.2^{a}$ & $11.1 \pm 0.5^{b}$ & $19.6 \pm 2.8^{a}$ \\
\hline Monoraphidium komarkova & $42.1 \pm 32.1^{b}$ & $20.4 \pm 12.8^{c}$ & $50.9 \pm 22.3^{\mathrm{a}}$ \\
\hline Oscillatoria sp. & & $20.0 \pm 5.0^{a}$ & \\
\hline Pediastrum clathratum & $4.0 \pm 1.0^{a}$ & $2.0 \pm 1.0^{a}$ & \\
\hline Pediastrum sp. & & $4.0 \pm 1.0^{a}$ & \\
\hline Pediastrum tetras & $12.0 \pm 0.5^{a}$ & & $6.0 \pm 2.0^{b}$ \\
\hline Pinnularia sp. & $1.0 \pm 0.0^{b}$ & $2.0 \pm 1.0^{a}$ & $3.5 \pm 2.5^{a}$ \\
\hline Rhizosolenia sp. & $1.5 \pm 1.5^{c}$ & $14.0 \pm 2.0^{a}$ & $6.5 \pm 2.2^{b}$ \\
\hline Rhizosolenia sp. & $2.0 \pm 1.0^{a}$ & $1.0 \pm 1.0^{a}$ & \\
\hline Scenedesmus acuminatus & $7.0 \pm 1.5^{b}$ & $6.0 \pm 2.0^{b}$ & $16.7 \pm 8.5^{a}$ \\
\hline Scenedesmus javanaensis & $22.4 \pm 0.5^{b}$ & $17.8 \pm 2.2^{b}$ & $24.6 \pm 0.2^{\mathrm{a}}$ \\
\hline Scenedesmus platydiscus & & $1.5 \pm 0.5^{b}$ & $11.0 \pm 2.4^{a}$ \\
\hline Scenedesmus quadricauda & $33.9 \pm 0.8^{a}$ & $33.1 \pm 1.0^{a}$ & $30.9 \pm 0.5^{a}$ \\
\hline Scenedesmus rectangularis & $1.0 \pm 1.0^{b}$ & $1.0 \pm 1.0^{b}$ & $14.0 \pm 2.0^{a}$ \\
\hline Scenedesmus securiformis & $30.2 \pm 10.2^{a}$ & $20.1 \pm 12.4^{b}$ & $27.7 \pm 16.4^{a}$ \\
\hline Staurastrum boreale & $4.0 \pm 2.0^{b}$ & $8.0 \pm 1.0^{a}$ & $2.0 \pm 1.0^{c}$ \\
\hline Staurastrum gracile & $2.5 \pm 1.5^{a}$ & $1.5 \pm 1.5^{a}$ & $2.0 \pm 1.0^{a}$ \\
\hline Staurastrum sp. & $4.5 \pm 1.2^{b}$ & $3.2 \pm 2.5^{b}$ & $8.0 \pm 2.0^{a}$ \\
\hline Staurodesmus cuspidatus & $1.0 \pm 1.0^{a}$ & $1.5 \pm 1.5^{a}$ & $2.0 \pm 1.0^{a}$ \\
\hline Staurodesmus extensus & $1.5 \pm 1.5^{a}$ & $1.5 \pm 1.5^{a}$ & $1.5 \pm 1.0^{a}$ \\
\hline Stauroneis sp. & $8.3 \pm 2.5^{a}$ & $9.4 \pm 3.2^{a}$ & $2.4 \pm 1.2^{a}$ \\
\hline Tetraedron caudatum & & $2.0 \pm 1.0^{a}$ & \\
\hline Treubaria sp. & $3.0 \pm 1.5^{a}$ & $4.0 \pm 2.0^{a}$ & $1.0 \pm 1.0^{b}$ \\
\hline \multirow[t]{2}{*}{ Ulothrix sp. } & $2.0 \pm 1.0^{a}$ & $2.0 \pm 1.0^{a}$ & $5.0 \pm 2.5^{a}$ \\
\hline & $11.9 \pm 13.9^{a}$ & $9.9 \pm 9.4^{a}$ & $13.1 \pm 11.2^{a}$ \\
\hline
\end{tabular}

Letras diferentes en la misma fila, indica diferencia significativa $(p<0.05)$.

Índices ecológicos. Los índices ecológicos evaluados en las comunidades planctónicas durante el cultivo se describen en la tabla 5. Los mayores valores del índice de Shannon-Wiener se registraron en el T1 (2.5-3.2 bits $/ \mathrm{ml})$ y T2 (2.4-3.2 bits/ml) con diferencia estadística $(\mathrm{p}<0.05)$ referente al T3 (1.8-2.8 bits $/ \mathrm{ml})$; con excepción de las semanas $9,10,14$ y 16 ; en las cuales no se presentó diferencia estadística $(p>0.05)$. El índice de uniformidad de Pielou fue mayor en el T1 (0.8-0.9) y T2 (0.8-0.7) las dos primeras semanas de cultivo; en las semanas 9,10 y 15 se registraron los menores valores de este índice (0.6-0.7) en el $\mathrm{T} 1$; el resto de semanas no se observó diferencia significativa en este índice entre los tratamientos. Con excepción de la semana 6 y 12 de cultivo la dominancia no presentó diferencia entre los tratamientos fluctuando entre 0.7 y 0.9 ( $p>0.05)$.

Tabla 5. Índices ecológicos de los microorganismos planctónicos asociadas al cultivo de $P$. magdalenae con tecnología biofloc.

\begin{tabular}{|c|c|c|c|c|c|c|c|c|c|}
\hline \multirow[t]{2}{*}{ SC } & \multicolumn{3}{|c|}{$\begin{array}{l}\text { Diversidad de } \\
\text { Shannon-Wiener } \\
\text { (bits/ind) }\end{array}$} & \multicolumn{3}{|c|}{ Uniformidad de Pielou } & \multicolumn{3}{|c|}{$\begin{array}{l}\text { Dominancia de } \\
\text { Simpson }\end{array}$} \\
\hline & T1 & $\mathrm{T} 2$ & T3 & & T & T3 & T1 & 2 & T3 \\
\hline 0 & $\begin{array}{l}3.0 \\
\pm 0.0^{a 12}\end{array}$ & $\begin{array}{c}2.9 \\
2 \pm 0.0^{a 1}\end{array}$ & $\begin{array}{c}2.0 \\
\pm 0.0^{b 2}\end{array}$ & $\begin{array}{c}0.8 \\
\pm 0.2^{\mathrm{a} 1}\end{array}$ & $\pm 0.2^{\mathrm{a}-1}$ & $\begin{array}{c}0.6 \\
\pm 0.2^{b 2}\end{array}$ & $\begin{array}{c}0.8 \\
\pm 0.2^{a 1}\end{array}$ & $\begin{array}{ll} & 0.8 \\
1 & \pm 0.2^{a 1}\end{array}$ & \\
\hline 1 & $\begin{array}{l}3.1 \\
\pm 0.0^{a 12}\end{array}$ & $\begin{array}{c}3.2 \\
2 \pm 0.0^{a t}\end{array}$ & & & & & & & \\
\hline 2 & & & & & & & & & \\
\hline 3 & $\begin{array}{c}3.5 \\
\pm 0.1^{\text {a1 }}\end{array}$ & $\begin{array}{c}2.5 \\
\pm 0.1^{b 2}\end{array}$ & $\begin{array}{c}2.5 \\
\pm 0.1^{b 1}\end{array}$ & $\begin{array}{c}0.8 \\
\pm 0.0^{\mathrm{a} 1}\end{array}$ & $\begin{array}{c}0.8 \\
\pm 0.0^{a 1}\end{array}$ & $\begin{array}{c}0.8 \\
\pm 0.0^{a} 1\end{array}$ & $\begin{array}{r}0 . \varepsilon \\
\pm 0.2\end{array}$ & $\begin{array}{c}0.8 \\
1+0.1^{a 1}\end{array}$ & \\
\hline 4 & & & & & & & & & \\
\hline 5 & & & & & & & & & \\
\hline 6 & & & $\begin{array}{r}1 \\
\pm 0\end{array}$ & & & & & & \\
\hline 7 & $\begin{array}{r}2.8 \\
\pm 0.5\end{array}$ & $\begin{array}{c}2.7 \\
\pm 0.5^{a 2}\end{array}$ & $\begin{array}{c}1.8 \\
\pm 0.5^{b 2}\end{array}$ & & & & $\begin{array}{c}0.8 \\
\pm 0.1^{a 1}\end{array}$ & & \\
\hline 8 & $\begin{array}{r}2.8 \\
\pm 0.8\end{array}$ & & & & & & $\begin{array}{r}0.5 \\
\pm 0 .\end{array}$ & & \\
\hline 9 & & & & & & & & & \\
\hline 10 & $\begin{array}{c}2.8 \\
\pm 0.2^{\mathrm{a} 2}\end{array}$ & $\begin{array}{c}2.8 \\
\pm 0.2^{a 1}\end{array}$ & $\begin{array}{c}2.8 \\
\pm 0.2^{a 1}\end{array}$ & & & $\begin{array}{c}0.9 \\
\pm 0.2^{a 1}\end{array}$ & $\begin{array}{c}0.8 \\
\pm 0.2^{\mathrm{a} 1}\end{array}$ & & \\
\hline 11 & $\begin{array}{l}3.9 \\
\pm 0.2^{\text {a1 }}\end{array}$ & $\begin{array}{l}2.6 \\
\pm 0.2^{b 2}\end{array}$ & $\begin{array}{c}1.9 \\
\pm 0.2^{c 2}\end{array}$ & & & $\begin{array}{l}0.7 \\
\pm 0.0^{b 2}\end{array}$ & $\begin{array}{c}0.9 \\
\pm 0.1^{a 1}\end{array}$ & 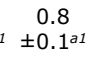 & \\
\hline 12 & $\begin{array}{r}3.1 \\
\pm 0.0^{\mathrm{a}}\end{array}$ & & & & & & & & \\
\hline 13 & & & & & & & & & \\
\hline 14 & $\begin{array}{c}2.6 \\
\pm 0.11^{\mathrm{a} 2}\end{array}$ & & & & & & & $\begin{array}{c}0.8 \\
1 \\
\pm 0.2^{a 1}\end{array}$ & \\
\hline 15 & $\begin{aligned} & 2.6 \\
\pm & 0.0^{\mathrm{a} z}\end{aligned}$ & $\begin{array}{c}2.2 \\
\pm 0.0^{b 3}\end{array}$ & & $\begin{array}{c}0.7 \\
\pm 0.1^{b 1}\end{array}$ & & & $\begin{array}{c}0.9 \\
\pm 0.1^{a 1}\end{array}$ & $\begin{array}{c}0.9 \\
1 \pm 0.1^{a 1}\end{array}$ & \\
\hline 16 & & $\begin{array}{c}2.5 \\
\pm 0.0^{\mathrm{a} 2}\end{array}$ & & & & $\begin{array}{l}0.8 \\
\pm 0.2^{\mathrm{a} 1}\end{array}$ & $\begin{array}{l}0.8 \\
\pm 0.2^{a 1}\end{array}$ & $\begin{array}{c}0.8 \\
1 \pm 0.1^{a 1}\end{array}$ & \\
\hline
\end{tabular}

SC: Semanas de cultivo;

Letras distintas en la misma fila indica diferencia significativa $(P<0.05)$ entre tratamientos para cada semana de cultivo. Números distintos en la misma columna indican diferencia estadística dentro de cada tratamiento $(p<0.05)$.

Caracterización bacteriana. En el inóculo inicial se identificaron 8 grupos (Tabla 6), con colonias bacterianas de la familia Enterobacteriaceae como Escherichia coli, Enterobacter sp., Klebsiella sp., Salmonella sp., caracterizadas como bacilos gramnegativos en concentraciones de $2.0 \times 10^{2}$ a $22.0 \times 10^{7} \mathrm{UFC} / \mathrm{ml}$. Del grupo de los bacilos grampositivos esporulados, se identificaron Bacillus subtilis y Bacillus sp., guardando relación con la especie Lactobacillus sp. También fueron identificadas bacterias descritas funcionalmente como heterotróficas, sulfitoreductoras, cocos y fijadoras de nitrógeno. 
Tabla 6. Grupos bacterianos aislados en el periodo de estabilización y maduración del inóculo de floc utilizado en el cultivo de $P$. magdalenae en sistema biofloc.

\begin{tabular}{|c|c|c|c|c|}
\hline Grupo & Especie & Forma & $\begin{array}{c}\text { Tinción de } \\
\text { Gram }\end{array}$ & $\mathrm{UFC} / \mathbf{m l}^{\mathbf{1}}$ \\
\hline \multirow[t]{2}{*}{ Heterótrofos totales } & & Bacilo & - & $8 \times 10^{5}$ \\
\hline & Escherichia coli & Bacilo & - & $5 \times 10^{6}$ \\
\hline \multirow{3}{*}{$\begin{array}{l}\text { Enterobacterias } \\
\text { (Enterobacteriaceae) }\end{array}$} & Enterobacter sp. & Bacilo & - & $22 \times 10^{7}$ \\
\hline & Klebsiella sp. & Bacilo & - & $2 \times 10^{2}$ \\
\hline & Salmonella sp. & Bacilo & - & $3 \times 10^{3}$ \\
\hline Sulfitoreductoras & Salmonella sp. & Bacilo & - & $3 \times 10^{3}$ \\
\hline \multirow{2}{*}{ Bacillus totales } & Bacillus subtilis & $\begin{array}{c}\text { Bacilo } \\
\text { esporulado }\end{array}$ & + & $12 \times 10^{6}$ \\
\hline & Bacillus sp. & $\begin{array}{c}\text { Bacilo } \\
\text { esporulado }\end{array}$ & + & $10 \times 10^{6}$ \\
\hline $\begin{array}{l}\text { Lactobacillus } \\
\text { totales }\end{array}$ & Lactobacillus sp. & Bacilo & + & $7 \times 10^{3}$ \\
\hline Vibrionaceae & Pseudomonas sp. & Bacilo & - & $12 \times 10^{3}$ \\
\hline \multirow{2}{*}{$\begin{array}{l}\text { Cocos } \\
\text { grampositivos }\end{array}$} & Micrococcus sp. & Cocos & + & $25 \times 10^{4}$ \\
\hline & Staphylococcus sp. & Cocos & + & $16 \times 10^{4}$ \\
\hline $\begin{array}{l}\text { Fijadores de } \\
\text { nitrógeno }\end{array}$ & & $\begin{array}{l}\text { Cocos y } \\
\text { Bacilos }\end{array}$ & $+/-$ & $20 \times 10^{6}$ \\
\hline
\end{tabular}

La caracterización cualitativa de los grupos bacterianos identificados (grupo/especie), en relación a su presencia/ ausencia, durante los cuatro meses de cultivo de $P$. magdalenae con tecnología biofloc se relacionan en la tabla 7. Además de los grupos presentes en el inóculo inicial, se encontró presencia de otras colonias de bacterias tales como Bacillus cereus, Vibrio cholerae y Vibrio sp. Las muestras de lodo del fondo, generado por la dinámica de cultivo, se caracterizaron por la presencia de la mayoría de los grupos previamente identificados con prevalencia de bacterias heterótrofas.

Tabla 7. Grupos bacterianos identificados en los diferentes tratamientos evaluados en el cultivo de $P$. magdalenae en fase de preengorde en sistema biofloc. +: Presencia, -: ausencia.

\begin{tabular}{ccccccccccc}
\hline & \multicolumn{3}{c}{$\begin{array}{c}\text { Día 15 de } \\
\text { cultivo }\end{array}$} & \multicolumn{3}{c}{$\begin{array}{c}\text { Día } \\
\text { cultivo }\end{array}$} & \multicolumn{3}{c}{$\begin{array}{c}\text { Día } \\
\text { cultivo de }\end{array}$} \\
\cline { 2 - 9 } & T1 & T2 & T3 & T1 & T2 & T3 & T1 & T2 & T3 \\
\hline Fijadoras de nitrógeno & - & + & - & - & + & + & - & + & + \\
Heterótrofas & + & + & + & + & + & + & + & + & + \\
Lactobacillus sp. & - & + & - & + & + & - & - & + & + \\
Bacillus cereus & - & + & - & - & + & - & - & + & + \\
Bacillus subtilis & + & + & + & + & + & + & + & + & + \\
Bacillus sp. & + & + & + & + & + & + & + & + & + \\
Pseudomonas sp. & + & + & - & + & - & - & + & - & - \\
Vibrio cholerae & - & + & - & + & + & - & - & + & + \\
Vibrio sp. & + & + & + & + & + & + & + & + & + \\
Escherichia coli & - & - & + & - & + & + & - & + & - \\
Klebsiella sp. & + & + & + & + & + & + & + & + & + \\
Salmonella sp. & - & - & - & + & - & + & + & + & + \\
Sulfitorreductoras & + & + & + & + & + & + & + & + & + & + \\
Enterobacter sp. & + & + & + & + & + & + & + & + & + \\
Micrococcus sp. & + & + & + & + & + & + & + & + & + & + \\
Staphylococcus sp. & + & - & - & - & - & + & - & - & + \\
\hline
\end{tabular}

La figura 1, muestra el número de UFC/ml de los grupos de bacterias identificadas a los 15, 45 y 90 días de cultivo. El T2 presentó el mayor número de UFC/ml con valores de $373.000 \mathrm{UFC} / \mathrm{ml}$ y $336.000 \mathrm{UFC} / \mathrm{ml}$; a los 15 y 90 días de cultivo respectivamente. La menor dominancia se registró en el T3 el día 15 (73.667 UFC/ml) y día 90 $(120.000 \mathrm{UFC} / \mathrm{ml})$, con diferencia estadística entre estos tratamientos $(p<0.05)$.

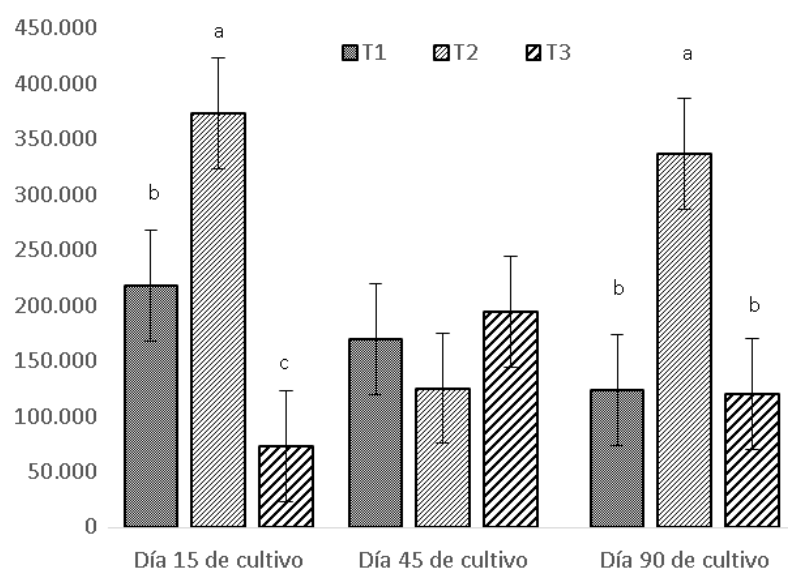

Figura 1. Número de bacterias (UFC/ml) a los 15,45 y 90 días de cultivo.

\section{DISCUSIÓN}

En el presente estudio, parámetros de calidad de agua (OD, temperatura y $\mathrm{pH}$ ) no presentaron diferencias entre los tratamientos; manteniéndose en los rangos considerados óptimos para los cultivos con tecnología biofloc $(6,8,16)$ y apropiados para el manejo de la especie en cultivo (17); asi mismo, la alcalinidad y la dureza se consideraron adecuadas para el mantenimiento y para la ruta de nitrificación de las bacterias propias del sistema $(9,4,12)$.

Los valores de TAN y $\mathrm{NH}_{3}$ fueron mayores a la más alta densidad (20 peces $/ \mathrm{m}^{3}$ ); posible consecuencia de la mayor cantidad de alimento ofrecida junto a una mayor proporción de materia orgánica en este tratamiento. En cultivos de tilapia con BFT se reportaron valores menores, entre 1.9 y 2.5 mg/l de TAN (13). Esto indica que los valores de TAN, deben ser estabilizados por la actividad bacteriana o por la acción conjunta entre las comunidades planctónicas y la microbiota, asimilando la fracción tóxica de estos compuestos e incorporándola a un reciclado eficiente de nutrientes. $\mathrm{El} \mathrm{NH}_{3}$, tóxico para los peces incluso a concentraciones muy bajas; se considera letal en cultivos comerciales por encima de $1.5 \mathrm{mg} / \mathrm{l}$ $(2,8,18)$, siendo los niveles aceptables por debajo de $0.5 \mathrm{mg} / \mathrm{l}$ (19). En el presente estudio, los niveles de $\mathrm{NH}_{3}$ fueron similares a los reportados por diversos autores, al evaluar el comportamiento y dinámica del sistema biofloc en el rendimiento en cultivo de peces y camarones, en su mayoría con valores no superiores a los $3.0 \mathrm{mg} / \mathrm{L}$ $(9,20,21)$.

En los cultivos BFT se presentan altos niveles de compuestos nitrogenados (TAN, $\mathrm{NH}_{3}$ ); pero su toxicidad depende de las condiciones de manejo dadas al sistema y del grado de tolerancia de la especie cultivada. En el 
presente estudio, a pesar de encontrarse por encima de valores óptimos reportados para el manejo de los cultivos BFT con otras especies $(2,20,21)$, los niveles de estos compuestos no incidieron en el manejo del mismo y el bienestar de la especie en cultivo; lo cual sugiere la tolerancia de la especie a las concentraciones registradas de estos compuestos nitrogenados. En la literatura no se encontraron reportes de dosis letal media (LC50) para el bocachico; por tanto se hace necesario estudiar la tolerancia de esta especie a estos compuestos nitrogenados.

Los valores de nitritos se mantuvieron similares en todos los tratamientos; mientras que los nitratos a mayor densidad (T2 y T3) fueron más altos; este comportamiento puede ser atribuido al manejo dado en función de la densidad establecida, en donde la ración alimenticia y por ende la carga de materia orgánica fue mayor en T3. De manera general, la calidad de agua del cultivo está asociada a las condiciones de manejo del sistema, siendo algunos parámetros afectados, en particular, los compuestos nitrogenados por las densidades evaluadas. Es importante señalar que, el hábito alimentario del bocachico (iliófago-detritivoro) es un factor a considerar para la adaptación este sistema de cultivo a especies con este hábito.

La composición de las comunidades plantónicas, asociadas al cultivo de $P$. magdalenae en sistema biofloc, sugiere un sistema de cultivo rico en microorganismos de importancia biológica y nutricional, con flujo de nutrientes y disponibilidad de alimento primario. Se identificaron seis grupos planctónicos (sin incluir bacterias) asociados a los macroagregados de floc, como son microalgas, rotíferos, ciliados, cladóceros, protista y copépodos, con mayor abundancia de este último grupo en el periodo de estabilización del inóculo; y rotífero, protista y ciliados durante el cultivo. Estos resultados concuerdan con los reportes de otros autores que han caracterizado las comunidades plantónicas de los macroagregados durante la conformación y estabilización del inóculo, así como en el desarrollo de cultivos con esta tecnología $(8,12,15)$.

La abundancia de los grupos de microorganismos estuvo relacionada con la densidad de siembra; por lo cual se puede inferir cierta presión de la especie sobre la abundancia de los microrganismos presentes, en función de la densidad de peces evaluadas y el tiempo de estabilización del sistema, tal como fue sugerido por Ballester et al (22) y Miaca et al (23), quienes consideraron que los elementos que producen los flóculos, tales como la fuente de carbono y el alimento balanceado, así como los peces acondicionados para el sistema, pueden tener una influencia directa sobre los grupos de organismos que se desarrollan y su abundancia en relación al tiempo de cultivo.

En el presente estudio, se observó la resuspensión de sólidos en la columna de agua; esto se sugiere como consecuencia del comportamiento y habito alimentario de la especie estudiada (bentónico - iliófagodetrítivoro); fenómeno que se ha observado en peces de comportamiento similar (24); los cuales, causan fuerte agitación en el fondo, incrementando la turbidez y facilitando el flujo de nutrientes a través de la cadena trófica (25); aunque una resuspensión excesiva podría afectar la productividad primaria; es posible que, a 20 peces $/ \mathrm{m}^{3}$ (T3) se causara una presión de pastoreo excesiva sobre la generación y ciclos reproductivos de los microorganismos.

En contexto, en los cultivos con BFT, la interacción y dominancia de las comunidades planctónicas y bacterianas, se presentan como un aporte significativo de proteína y energía para las especies de interés del cultivo, al contribuir en el flujo de nutrientes mediante las cadenas tróficas establecidas desde los productores primarios a los consumidores secundarios, de preferencia por consumo de microalgas y bacterias mediante el pastoreo continuo en los macroagregados de floc $(12,26)$.

Los protistas fueron el grupo de mayor densidad (Tabla 3). La importancia de este grupo radica en la eliminación de contaminantes, especialmente de compuestos nitrogenados, contribuyendo a la formación de bioagregados y flóculos (27), en la depredación de poblaciones bacteriana que pueden llegar a ser patógenas (28) y en la formación, distribución y composición de la comunidad bacteriana (27).

El grupo de los rotíferos constituye otro de los grupos representativos en las cadenas tróficas presentes en los ambientes de cultivo con tecnología biofloc (12). En el presente estudio, los rotíferos fueron el segundo grupo de mayor densidad; la cual osciló en el rango reportado (5 a $196 \mathrm{ind} / \mathrm{ml}$ ) por estudio similares $(12,15)$. Asociados en igual manera a la dinámica del sistema y con la formación de los macroagregados, se encuentran los ciliados (Vorticellas sp. y Paramecio sp.), también caracterizados en los cultivos de tilapia y camarón desarrollados con tecnología biofloc $(15,23,26)$.

De las especies menos abundantes, se resalta la presencia de Monhytera similis dentro del grupo de los anélidos, grupo considerado de gran importancia dentro del sistema biofloc por presentar altos contenidos de proteína cruda y ácidos grasos esenciales; así como, especies del grupo de las amebas, cladóceros y copépodos. Entre las microalgas los géneros Ankistrodesmus y Scenedesmus (clorófita), fueron predominantes en el grupo de las microalgas, referenciado en ocasiones por la cantidad y calidad de nutrientes, y el consumo selectivo de ciertas especies de ciliados y rotíferos que controlan las poblaciones microalgales (12).

La caracterización de los grupos de microrganismos descritos, es frecuente en la mayoría de los estudios de cultivo con BFT $(12,15,16,22,26)$. Por lo cual es posible sugerir el establecimiento de una comunidad planctónica específica, dominante, uniforme y diversa, con influencia de características como estrategia reproductiva, tamaño pequeño, ciclo de vida corto y amplia tolerancia a factores ambientales, por parte de los microorganismos; al igual que, por condiciones del sistema como fuente de carbono, inóculo inicial; y como en el presente estudio, por el comportamiento y habito alimenticio de la especie cultivada.

La diversidad de los microorganismos encontrados, mostró rango máximo de 3.9 bits/ind, con alta dominancia $(>0.7)$ e uniformidad (>0.6). Al establecerse por grupo identificado, los rotíferos y microalgas fueron los grupos con mayor riqueza taxonómica y abundancia durante el cultivo. Estos resultados permiten inferir que un índice de baja diversidad, puede indicar que existen pocas especies en relación al total de individuos caracterizados en los 
sistemas BFT. De manera general los sistemas BFT, distan de ser un sistema de producción cerrada y con utilización de inóculos seleccionados, bajo lo cual es predecible el patrón de microrganismos y comunidades planctónicas asociadas a los macroagregados de floc.

En relación a la microbiota presente y con incidencia directa en la dinámica de nutriente del sistema, la caracterización de bacterias del tipo Enterobacteriaceae, Bacillus, Lactobacillus, Heterotróficas, Sulfitoreductoras, Cocos y Fijadoras de nitrógeno, coinciden con los reportes de Monroy-Dosta et al (15). Estos autores determinaron dentro de comunidad microbiana asociada al biofloc en un cultivo de tilapia, géneros de bacterias heterotróficas como Pseudomonas, Bacillus, Vibrios, Enterobacter y Micrococcus.

Este tipo de bacterias en conjunto, promueven rutas definidas en la eliminación de compuestos nitrogenados tóxicos en los sistemas de cultivo. Ebeling et al (4), definen la eliminación de nitrógeno por algas fotoautotróficas, la inmovilización por bacterias heterótrofas de la biomasa microbiana proteica y la oxidación quimio-autótrofa en nitrato por bacterias nitrificantes, como las principales rutas dentro del flujo de nutrientes en los sistemas de cultivo BFT. La importancia relativa de cada uno varía según el tipo y la intensidad del sistema de producción. En el presente estudio, a pesar de estimular la inmovilización de las bacterias heterótrofas, el sistema estuvo dominado por bacterias nitrificantes.
Las comunidades microbianas en sistemas BFT, se presentan de manera diversa incluyendo bacterias y patógenos oportunistas; así como, bacterias neutrales y beneficiosas. Al enfatizar en algunos de los grupos identificados, se resalta la presencia de colonias de los géneros Bacillus y Lactobacillus, con características probióticas en el desarrollo de cultivo de peces, principalmente por secretar una gran variedad de exoenzimas y polímeros que generan un ambiente hostil a bacterias patógenas (29). En general se resalta el mantenimiento del sistema por las comunidades bacterianas establecidas, mediante rutas de nitrificación y reciclado de compuestos nitrogenados, bajo condiciones mínimas de nutrientes y el manejo de la calidad de agua en rangos adecuados para el cultivo de la especie.

Los resultados del presente estudio permiten concluir que la composición de las comunidades planctónicas y bacterianas fue similar en todos los tratamientos, siendo los grupos más abundante rotífero, protistas y ciliados; las cuales son una fuente de alimento para bocachico. Las bacterias presentes, se relacionan directamente con la dinámica del sistema, con mayor cantidad de enterobacterias y heterotróficas.

\section{Conflicto de intereses.}

Los autores del presente artículo declaramos no tener ningún conflicto de intereses que ponga en riesgo su publicación.

\section{REFERENCIAS}

1. Vinatea L, Gálvez A, Browdy C, Stokes A, Venero J, Haveman J, Lewis B, Lawson A, Shuler A, Leffler $G$. Photosynthesis, water respiration and growth performance of Litopenaeus vannamei in a super-intensive raceway culture with zero water exchange: interaction of water quality variables. Aquacultural Engineering 2010; 42(1):17-24. DOI: https://doi.org/10.1016/j.aquaeng.2009.09.001

2. Avnimelech Y. Biofloc Technology - A Practical Guidebook. The World Aquaculture Society; 2009. https://books.google.com.co/books/about/Biofloc Technology.html?id=FI-CrgEACAAJ\&redir_esc $=y$

3. Wasielsky J, Atwood H, Stokes A, Browdy C. Effect of natural production in a zero exchange suspended microbial floc based super-intensive culture system for white shrimp Litopenaeus vannamei. Aquaculture 2006; 258:396-403. DOI: https://doi. org/10.1016/j.aquaculture.2006.04.030

4. Ebeling J, Timmons M, Bisogni J. Review of autotrophic and heterotrophic bacterial control of ammonianitrogen in zero-exchange production systems: stoichiometry and experimental verification. Aquaculture 2006; 257:346-358. DOI: https://doi. org/10.1016/j.aquaculture.2006.03.019

5. Avnimelech Y. Feeding with microbial flocs by tilapia in minimal discharge bio-flocs technology ponds. Aquaculture 2007; 264:140-147. DOI: https://doi. org/10.1016/j.aquaculture.2006.11.025
6. Ray A, Lewis B, Browdy C, Leffler J. Suspended solids removal to improve shrimp Litopenaeus vannamei, production and an evaluation of a plant based feed in minimal-exchange, superintensive culture systems. Aquaculture 2010a; 299:89-98. DOI: https://doi. org/10.1016/j.aquaculture.2009.11.021

7. Ekasari J, Crab R, Verstraete W. Primary nutritional content of Bio-flocs cultured with different organic carbon sources and salinity. HAYATI Journal of Biosciences. 2010; 17(3):125-130. DOI: https:// doi.org/10.4308/hjb.17.3.125

8. Crab R, Defoirdt T, Bossier P, Verstraete $W$. Biofloc technology in aquaculture: Beneficia effects and future challenges. Aquaculture 2012; (356-357):351-356. https://doi.org/10.1016/j. aquaculture.2012.04.046

9. Azim M, Littlea D. The biofloc technology (BFT) in indoor tanks: Water quality, biofloc composition, and growth and welfare of Nile tilapia Oreochromis niloticus. Aquaculture 2008; 283(1-4):29-35. https://doi.org/10.1016/j. aquaculture.2008.06.036

10. Kunh D, Lawrence A, Boardman G, Patnaik S, Marsh L, Flick G. Evaluation of two types of bioflocs derived from biological treatment of fish effluent as feed ingredients for Pacific white shrimp, Litopenaeus vannamei. Aquaculture 2010; 303(1-4):28-33. https://doi.org/10.1016/j. aquaculture.2010.03.001 
11. Suita S, Ballester E, Abreu P, Wasielesky WJr. Dextrose as carbon source in the culture of Litopenaeus vannamei (Boone, 1931) in a zero exchange system. Latin American Journal of Aquatic Research 2015; 43(3): 526-533. http://www.lajar. cl/pdf/imar/v43n3/Articulo $43 \quad 3$ 13.pdf

12. Ray A, Seaborn G, Leffler J, Wilde S, Lawson A, Browdy C. Characterization of microbial communities in minimal-exchange, intensive aquaculture systems and the effects of suspended solids management. Aquaculture 2010b; 310:130-138. DOI: https:// doi.org/10.1016/j.aquaculture.2010.10.019

13. Kubitza F. Criacão de tilapia em sistema com bioflocos sem renovacão de água. Panorama da Aqüicultura 2011; 14-23. URL Disponible en http:// www.acquaimagem.com.br/docs/Pan125 Kub bioflocos piscicultura.pdf .

14. Aladro-Lubel M. Manual de protozoarios [Manual of protozoa]. Faculty of Sciences, Autonomous University of Mexico, Mexico 2009; p 123

15. Monroy-Dosta M, De Lara-Andrade R, CastroMejia J, Castro-Mejia G, Coelho-Emerenciano M. Composición y abundancia de comunidades microbianas asociadas al biofloc en un cultivo de tilapia. Rev Biol Mar Oceanogr. 2013 . 48(3):511-520. http://dx.doi.org/10.4067/S071819572013000300009

16. Emerenciano M, Gaxiola G, Cuzon G. Biofloc Technology (BFT): A Review for Aquaculture. Application and Animal Food Industry. INTECH 2013; 12:301-327. DOI: http://dx.doi. org/10.5772/53902

17. Garcia JJ, Celis LM, Villalba EL, Mendoza LC, Brú SB, Atencio VJ, Pardo SC. Evaluation of the bocachico polyculture Prochilodus magdalenae and Oreochromis niloticus tilapia using perifiton fixing surfaces. Rev Fac Med Vet Zoot. 2011; 58(2):71-83. https://revistas.unal.edu.co/index.php/remevez/ article/view/25398

18. Hargreaves J. Biofloc Production Systems for Aquaculture. SRAC 2013; 4503:8-10. https:// aquaculture.ca.uky.edu/sites/aquaculture.ca.uky. edu/files/srac 4503 biofloc production systems for_aquaculture.pdf

19. Pérez-Fuentes J, Hernández-Vergara $M$, Pérez-Rostro $\mathrm{C}$, Fogel I. C:N ratio affect nitrogen removal and production of nile tilapia Oreochromis niloticus raised in a biofloc system under high density cultivation. Aquaculture 2016; 452:247-251. https://doi. org/10.1016/j.aquaculture.2015.11.010

20. Schveitzer R, Arantes R, Costódio P, Espírito-Santo C, Arana LV, Seiffert W, Andreatta E. Effect of different biofloc levels on microbial activity, water quality and performance of Litopenaeus vannamei in a tank system operated with no water exchange. Aquacultural Engineering 2013; 56:59-70. https:// doi.org/10.1016/j.aquaeng.2013.04.006
21. Lorenzo M, Souza E, Schledera D, Rezendea $P$, Seifferta W, Vieiraa F. Intensive hatchery performance of Pacific white shrimp in the biofloc system under three different fertilization levels. Aquacultural Engineering 2016; 72-73:40-44. https://doi.org/10.1016/j.aquaeng.2016.04.001

22. Marrugo-Negrete J, Navarro-Frómeta A, RuizGuzman J. Total mercury concentrations in fish from Urrá reservoir (Sinú river, Colombia). Six years of monitoring. Rev MVZ Córdoba 2015; 20(3): 47544765. https://doi.org/10.21897/rmvz.45

23. Ballester E, Abreu P, Cavalli R, Emerenciano M, Abreu $L$, Wasielesky W. Effect of practical diets with different protein levels on the performance of Farfantepenaeus paulensis juveniles nursed in a zero exchange suspended microbial flocs intensive system. Aquaculture Nutrition 2010; 16: 163-172. https://doi.org/10.1111/j.13652095.2009.00648.x

24. Maicá P, Borba M, Wasielesky W. Effect of low salinity on microbial floc composition and performance of Litopenaeus vannamei (Boone) juveniles reared in a zero water-exchange super-intensive system. Aquaculture Research 2012; 43:361-370. https:// doi.org/10.1111/j.1365-2109.2011.02838.x

25. Della Rosa P, Ortiz J, Cáceres A, Sánchez S, Roux J. Desempeño del sábalo Prochilodus lineatus en policultivo con pacú Piaractus mesopotamicus. Lat Am J Aquat Res 2016; 44(2):336-341. http:// dx.doi.org/10.3856/vol44-issue2-fulltext-14.

26. Milstein A, Ahmed AF, Masud OA, Kadir A, Wahab MA. Effects of the filter feeder silver carp and the bottom feeder's mrigal and common carp on small indigenous fish species (SIS) and pond ecology. Aquaculture 2006; 258 (1-4): 439-451. https:// doi.org/10.1016/j.aquaculture.2006.04.045

27. Loureiro K, Wilson W, Abreu P. Use of protozoa, rotifers and nematodes as live feed for shrimp cultured in the BFT system. Atlântica Rio Grande2012; 34(1):5-12. https://doi.org/10.5088/ atl.2012.34.1.5

28. Abreu C, Ballester E, Odebrecht C, Wasielesky WJr, Cavalli R, Granéli W, Anésio A. Importance of biofilm as food source for shrimp (Farfantepenaeus paulensis) evaluated by stable isotopes. J Exp Mar Bio Ecol. 2007; 347 (1-2):88-96. https://doi. org/10.1016/j.jembe.2007.03.012

29. Wu L, Peng $C$, Peng $Y$, Li L, Wang S, Ma Y Effect of wastewater $\mathrm{COD} / \mathrm{N}$ ratio on aerobic nitrifying sludge granulation and microbial population shift. Journal Environment Science. 2012; 24(2):234-241. https://doi.org/10.1016/S1001-0742(11)60719-5

30. Monroy D, Castro B, Fernández $P$, Mayorga R. Inhibition of Aeromonas hydrophila by probiotic strains isolated from the digestive tract of Pterophyllum scalare. Rev Mex Ing Quím 2010; 9(1):37-42. http://rmiq.org/iqfvp/Pdfs/Vol9\%20 no\%201/RMIQ_Vol9_No_1_5.pdf 